\title{
Remark to my previous paper on a bilateral Tauberian theorem
}

\author{
By Åke Pleijel
}

This remark is directly connected with paper [1] to which we refer for notations and theorems.

When $s-h$ is an integer the conditions of the Abelian theorem $A(-\infty,+\infty)$ require more from $\sigma(\lambda)$ than is obtained in the conclusion of the Tauberian theorem $T(-\infty,+\infty)$. This makes it natural to ask whether $T(-\infty,+\infty)$ can be sharpened so as to give not only $\sigma(\lambda), \sigma(-\lambda) \in I^{s}$ for positive values of $\lambda$ but also

and

$$
\begin{array}{ll}
I^{-s}(\sigma(\lambda)+\sigma(-\lambda)) \in \omega^{0} & \text { when } s-h=\text { odd integer } \\
I^{-s}(\sigma(\lambda)-\sigma(-\lambda)) \in \omega^{0} & \text { when } s-h=\text { even integer. }
\end{array}
$$

Results of this kind were obtained in section 8 of [1] for the so-called non-exceptional cases. However, in the exceptional cases these results did not follow from the considerations in [1] but are, as will be seen, consequences of a lemma due to Hardy and Littlewood.

The main asymptotic condition in $T(-\infty,+\infty)$ is split into relations (8), (9) of [1]. Assume for instance that $s-h$ is an odd integer. According to the result of $T(-\infty,+\infty)$ we know that $\sigma(\lambda)$ and $\sigma(-\lambda) \in I^{s}$ so that also $S(\lambda)=\sigma(\lambda)+\sigma(-\lambda) \in I^{s}$ and $I^{-s / 2} S(\sqrt{\Lambda}) \in I^{0}$. Relation (9) of [1] can be written

$$
\int_{0}^{\infty} \Lambda^{-k}(\Lambda+T)^{-1} d I^{-s / 2} S(\sqrt{\Lambda})=T^{-1} P_{2}\left(T^{-1}\right)+o\left(|T|^{-k-1}\right),
$$

where $k=-\frac{1}{2} s+\frac{1}{2}(h-1)$ is a non-negative integer. In the way indicated in section 4 of [1] this relation can be replaced by

$$
\int_{0}^{\infty}(\Lambda+T)^{-1} d I^{-s / 2} S(\sqrt{\Lambda})=A T^{-1}+o\left(|T|^{-1}\right)
$$

where $A$ is a constant.

The lemma of Hardy and Littlewood reads (see [2], p. 198 for the simple proof)

If $t \int_{0}^{\infty}(\lambda+t)^{-1} d \varphi(\lambda) \rightarrow A$ when $t \rightarrow+\infty$ and if $\varphi \in I^{0}$ then $\varphi \in \omega^{0}$ and $\varphi(\lambda)-\varphi(0) \rightarrow A$ when $\lambda \rightarrow+\infty$.

Application of this lemma shows that $I^{-s / 2} S(\sqrt{\Lambda}) \in \omega^{0}$, i.e. $I^{-s}(\sigma(\lambda)+\sigma(-\lambda)) \in \omega^{0}$ for $\lambda \rightarrow+\infty$, provided $s-h$ is an odd integer. 


\section{ÅKE PLEIJEL, Bilateral Tauberian theorem}

The case when $s-h$ is an even integer is similarly treated with the help of relation (8) in [1]. The result is, that in this case $I^{-s}(\sigma(\lambda)-\sigma(-\lambda)) \in \omega^{0}$ for $\lambda \rightarrow+\infty$.

I take the opportunity to mention gratefully that my study of bilateral Tauberian theorems was supported by National Science Foundation Grant G-10098 during a stay at the University of California at Berkeley.

\section{RE F E R N C E S}

1. Pleijel, A., A bilateral Tauberian theorem. Arkiv för Matematik, 4, 561-571 (1962).

2. WIDder, D. V., The Laplace transform. Princeton University Press, 1946. 